\title{
LONG-TERM PHOTOMETRIC BEHAVIOR OF 18 CARBON STARS IN CYGNUS
}

\author{
A. Alksnis \\ Institute of Astronomy, University of Latvia, Raina bulv. 19, \\ Riga LV-1586, Latvia
}

Received October 15, 2003

\begin{abstract}
The large variety of long-term (secondary) variations is demonstrated on the basis of a 30 year long photographic red magnitude time-series for 18 carbon stars in Cygnus based on the observations carried out with the Baldone Schmidt telescope.
\end{abstract}

Key words: stars: carbon, variables

\section{INTRODUCTION AND DATA}

There are many long-period variables - Mira stars and SR-variables - among the later subclasses of carbon stars. The main cycle length or the primary period (of several hundred days) is due to pulsations of the outer layers of the star. In many cases this pulsational variation is superposed on variations of much longer time scales of unknown origin. Their study needs an homogeneous photometric long time-series, at least of several decades.

Soon after the 80/120/240 cm Schmidt telescope of the Radioastrophysical (now Astrophysical) Observatory at Baldone was put into operation, photometric monitoring of carbon stars was initiated, mainly in the red $R\left(\lambda_{0}=0.63 \mu \mathrm{m}\right)$ passband and, less frequently in passbands similar to the $B$ and $V$ of the $U B V$ system. $R\left(\lambda_{0}=0.63\right.$ $\mu \mathrm{m})$ passband is similar to that of the $R G U$ system of Becker; for a description of the system see Straižys (1977).

The region most intensively studied was the 240 sq. degree area in Cygnus, covering Galactic longitudes from 83.5 to $96.5^{\circ}$ and latitudes from $-9.5^{\circ}$ to $+9.5^{\circ}$ and comprising three Galactic longitude zones nearly $5^{\circ}$ wide - the $90^{\circ}$-zone (observed in 1971-1975 and less 


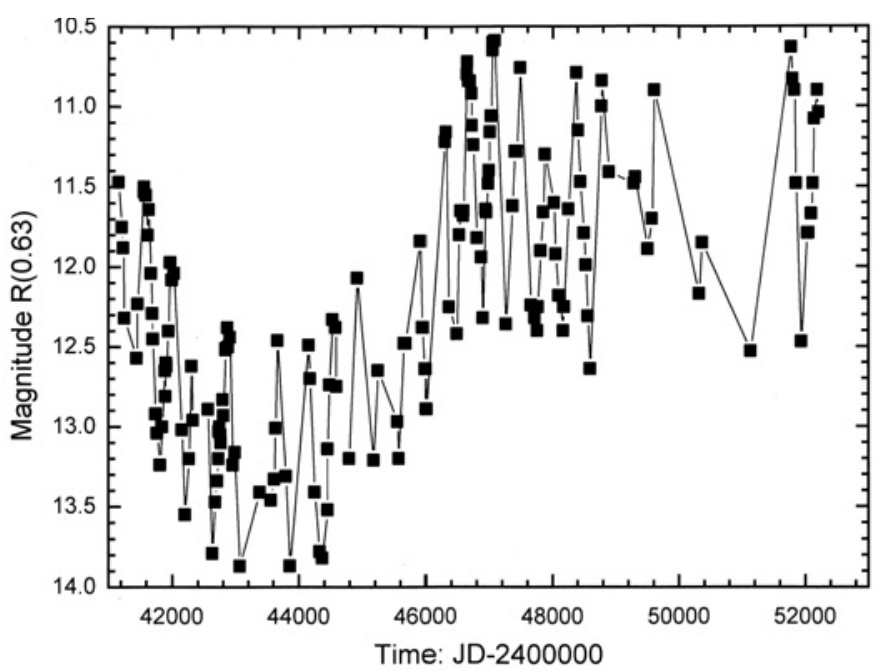

Fig. 1. Observed light curve of V1558 Cyg.

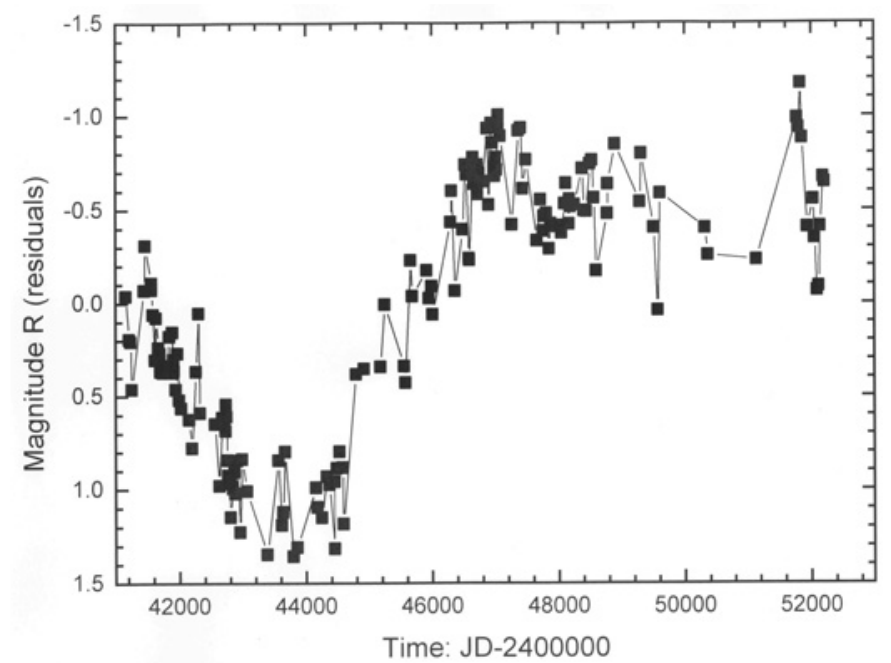

Fig. 2. Pre-whitened light curve of V1558 Cyg.

frequently in 1976-1984), the $86^{\circ}$-zone $(1972-1984)$ and the $94^{\circ}$ zone (1973-1984). 141 carbon stars identified on objective prism plates have been measured. The derived magnitudes and light curves of variable stars, together with their finding charts and comparison stars, have been published by Alksnis \& Alksne $(1977,1988)$. The analysis of the 10-12 year long time series revealed 45 long period variables (LPVs), while the other 96 stars were divided into 5 groups according to their range of variation (Alksnis \& Alksne 1988). About 20 stars, which exposed secondary variation (or long-term trend) 
were chosen for further monitoring from 1987 up to 2002. Now we have nearly 30 year long time series of the $R$ magnitudes of 18 carbon stars for examination of their long-term variations.

\section{DATA PROCESSING AND RESULTS}

The data are not evenly distributed over the 30 year time interval, there are considerable gaps in time series for some stars. The data were analyzed with a Fourier transform from the program TS of the American Association of Variable Star Observers (AAVSO), and the highest power periods were derived. The observed light curves were pre-whitened, i.e., sinewave corresponding to the pulsational variation was subtracted for better visualization of long-term changes. As an example, the observed curve and the pre-whitened curve for V1558 Cyg are shown in Figures 1 and 2, respectively.

In some cases, the first harmonic as a sinewave was also subtracted. The light fluctuations seen in Figure 2 and in other prewhitened light curves on time scales near or less of the pulsational period might be due to changes of the shapes and amplitudes of individual pulsational cycles, the changes of the pulsational period, temporal phase shifts and observational errors. We will consider them as noise when studying the long-term variations. In some cases, when a decrease or increase of the period was evident, two or more sinewaves were used for pre-whitening in different time intervals. In all figures the points of the adjacent observed values, as well as the residuals are connected with straight lines for visual aid only.

The sinewave periods and amplitudes are presented in Table 1. It contains star numbers from the General Catalog of Galactic Carbon Stars (Alksnis et al. 2001) and the General Catalogue of Variable Stars (Kholopov et al. 1998). The next columns give the period $P_{1}$ and full amplitude $A_{1}$ of the pulsational sinewave, the observed range in magnitude $R$ for the secondary variation, the period $P_{2}$ and half-amplitudes $A_{2}$ for sinewave of the secondary variation with the highest power, the period $P_{3}$ and half-amplitude $A_{3}$ for that with the second-highest power.

The long-term variations of the stars discussed are very different, several kinds of long-term light curves, however, can be noticed. 
Table 1. Parameters of the light curves of the studied carbon

\begin{tabular}{lcccccccc}
\multicolumn{2}{c}{ stars. } \\
\hline CGCS & GCVS & $\begin{array}{c}P_{1} \\
(\mathrm{~d})\end{array}$ & $\begin{array}{c}A_{1} \\
(\mathrm{mag})\end{array}$ & $\begin{array}{c}\text { Range } \\
(\mathrm{mag})\end{array}$ & $\begin{array}{r}P_{2} \\
(\mathrm{~d})\end{array}$ & $\begin{array}{c}A_{2} \\
(\mathrm{mag})\end{array}$ & $\begin{array}{c}P_{3} \\
(\mathrm{~d})\end{array}$ & $\begin{array}{c}A_{3} \\
(\mathrm{mag})\end{array}$ \\
\hline 5206 & V1554 Cyg & 333.7 & 1.50 & 0.5 & 40500 & 1.16 & 6110 & 0.19 \\
5218 & V1808 Cyg & 350.2 & 0.96 & 0.7 & 9800 & 0.35 & 5520 & 0.14 \\
5386 & V637 Cyg & 386.4 & 1.54 & 0.6 & 10900 & 0.34 & - & - \\
5289 & V1563 Cyg & 388.1 & 1.48 & 0.4 & 4900 & 0.15 & 420 & 0.10 \\
5416 & V1732 Cyg & 395.5 & 1.66 & 0.5 & 40400 & 0.68 & 7560 & 0.19 \\
4952 & V1523 Cyg & 396.1 & 1.50 & 1.1 & 14200 & 0.50 & 2570 & 0.30 \\
4964 & V1525 Cyg & 407.5 & 1.34 & 0.4 & 3400 & 0.07 & - & - \\
5271 & V1558 Cyg & 422.4 & 1.40 & 1.7 & 12300 & 0.70 & 6720 & 0.51 \\
5358 & V1426 Cyg & 474.6 & 1.74 & 2.0 & 9020 & 0.45 & 1620 & 0.11 \\
5390 & V1568 Cyg & 480.8 & 1.86 & 2.3 & 6100 & 0.73 & 2980 & 0.46 \\
5280 & V603 Cyg & 496.5 & 0.84 & 2.0 & 1240 & 0.62 & 3900 & 0.47 \\
5163 & V573 Cyg & 509.0 & 1.30 & 1.7 & 24900 & 1.64 & 3850 & 0.14 \\
5331 & V1556 Cyg & 509.1 & 1.22 & 1.3 & 45000 & 1.56 & 3910 & 0.29 \\
5057 & V1715 Cyg & 513.4 & 1.14 & 1.6 & 2290 & 0.41 & 1500 & 0.37 \\
5204 & V1555 Cyg & 516.8 & 0.92 & 1.7 & 40500 & 0.44 & 7840 & 0.31 \\
5404 & V644 Cyg & 516.8 & 1.30 & 2.3 & 17000 & 1.17 & 4064 & 0.27 \\
5431 & V2074 Cyg & - & - & 1.4 & 7560 & 0.21 & - & - \\
5192 & V1983 Cyg & - & - & 1.7 & - & - & - & - \\
\hline
\end{tabular}

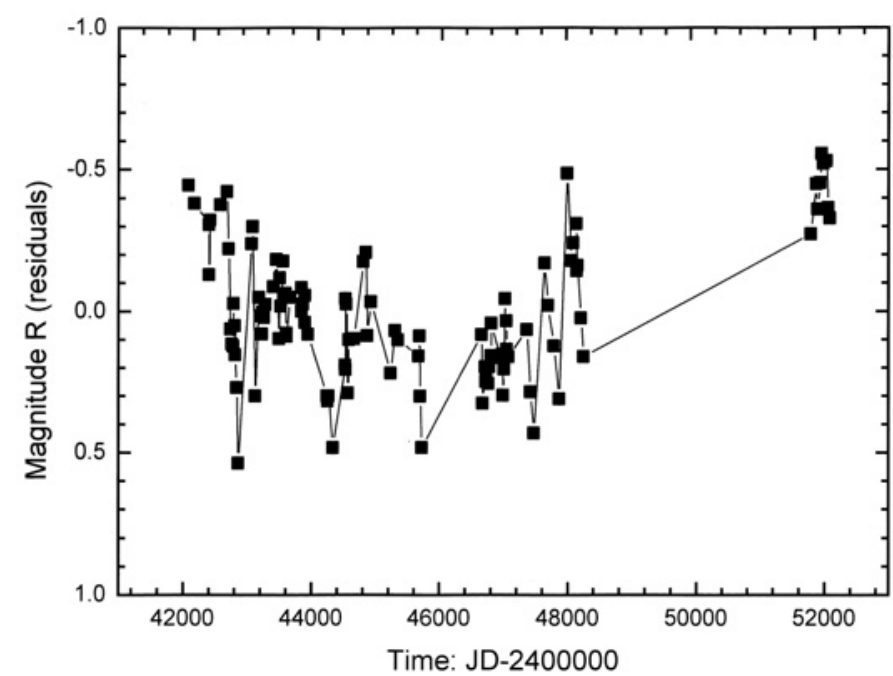

Fig. 3. Pre-whitened light curve of V637 Cyg.

Minor (0.4-0.7 mag range) smooth long-term variations have been detected in the stars V1554 Cyg, V1808 Cyg, V637 Cyg (Figure 3), V1563 Cyg, V1732 Cyg and V1525 Cyg. (Note that five of them have 
the shortest pulsational periods $P_{1}<400 \mathrm{~d}$ ). Sinewave approximations for oscillations, which have significant amplitude, have periods $>5000$ d. For V1554 Cyg the extrapolation to a longer time interval, however, would predict a range of $>2$ mag.

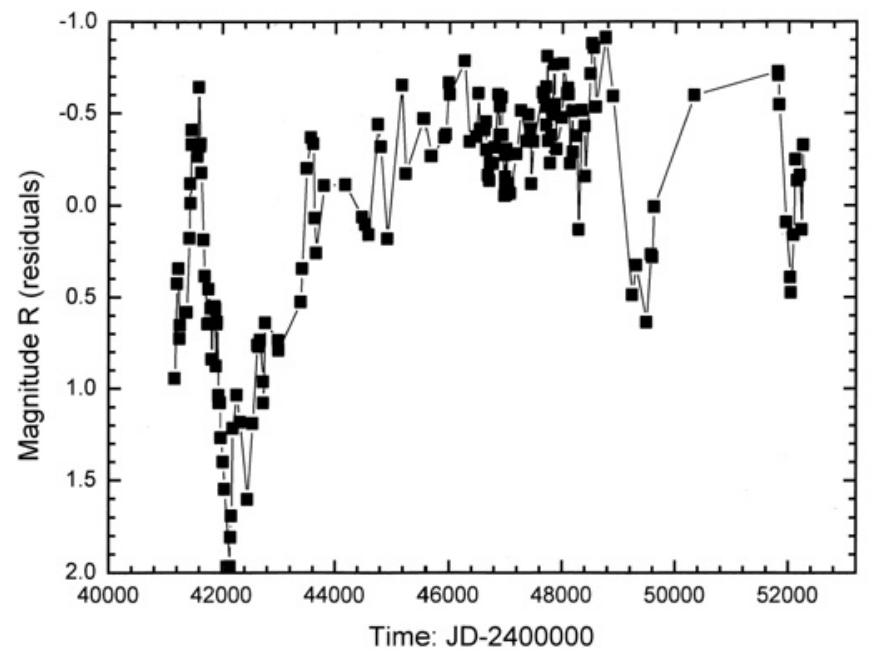

Fig. 4. Pre-whitened light curve of V1523 Cyg.

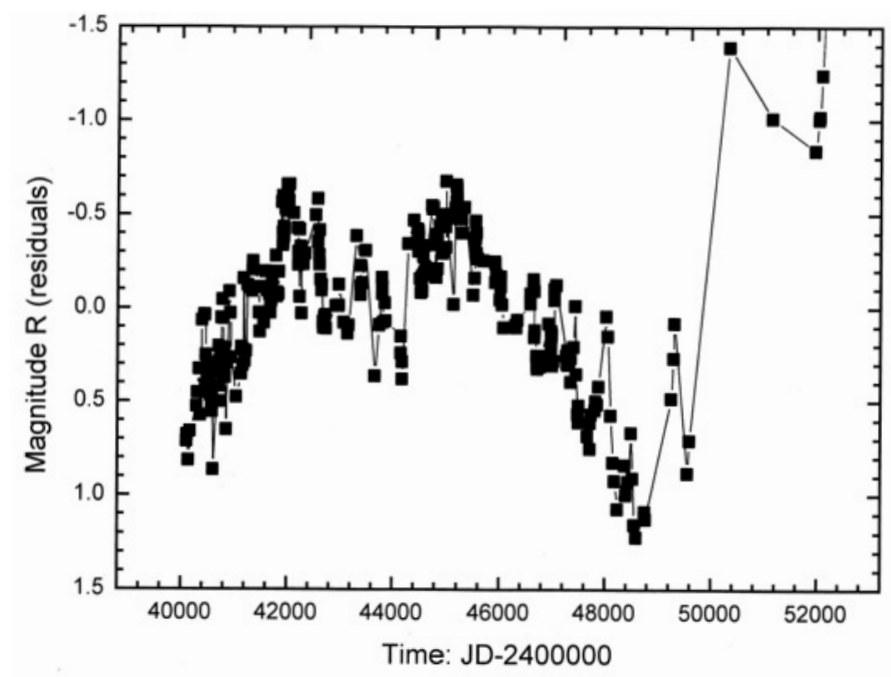

Fig. 5. Pre-whitened light curve of V1426 Cyg.

Two of the six stars - V1554 Cyg and V1808 Cyg - display rather sharp, about 1 mag deep and 2-3 $P_{1}$ long declines followed by a slower recovery. Two (possibly three) similar light drops underwent the star V1523 Cyg (Figure 4), which also has a pulsational period of $<400$ 
d. These light drops remind us the steep decline and recovery event found in the near infrared long-term variations of the carbon Mira star R For and suggested by Whitelock et al. (1997) to be caused by an increased dust obscuration.

A common feature for the light curves of V1426 Cyg (CIT 13) (Figure 5), V1568 Cyg and V1555 Cyg is a case of the brightening of the star by $2.5 \mathrm{mag}$ during the time interval of $3 P_{1}$. For the three stars the brightening starts after a long interval of slower light decrease, with different rate, however, for different stars. A short $\left(\approx 2 P_{1}\right)$ and shallow $(0.5-1.0 \mathrm{mag})$ dip is seen on the light curve at the time 9-12 $P_{1}$ before the start of the light rise. For V1568 Cyg the long-term decrease and high rise looks like a part of a cyclic oscillation with the $P_{2}=6100 \mathrm{~d}$ period (Figure 6). For V1556 Cyg the pre-whitened light curve is slightly similar to that of V1555 Cyg, but the high rise is absent.

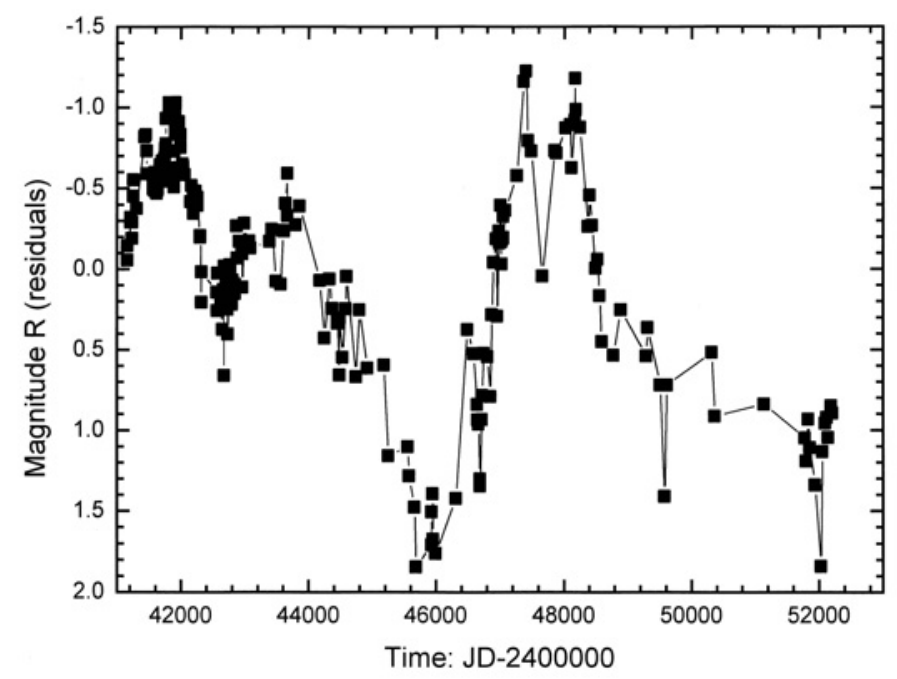

Fig. 6. Pre-whitened light curve of V1568 Cyg.

For V603 Cyg and V1715 Cyg the oscillations have a zigzag shape. The most expressed secular trend shows V644 Cyg - a monotonous decline of light with a rate of $0.08 \mathrm{mag} /$ year (Figure 7 ).

The stars V1558 Cyg (Figure 2), V573 Cyg and V644 Cyg (Figure 7 ) have smooth large amplitude (1.7-2.3 mag) secondary variation, which can be approximated by a sinewave of periods longer than $17000 \mathrm{~d}$. 


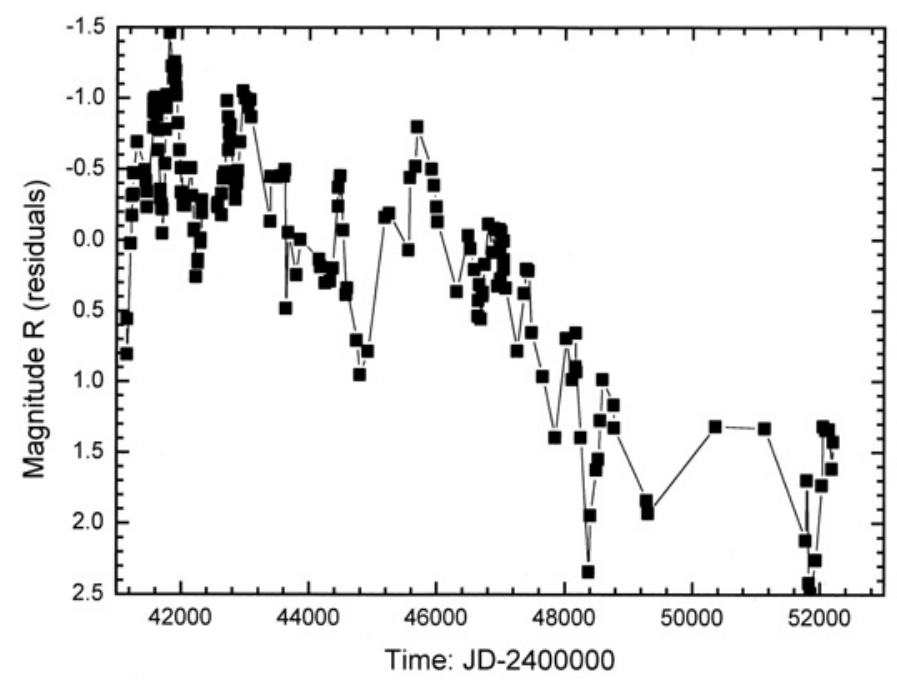

Fig. 7. Pre-whitened light curve of V644 Cyg.

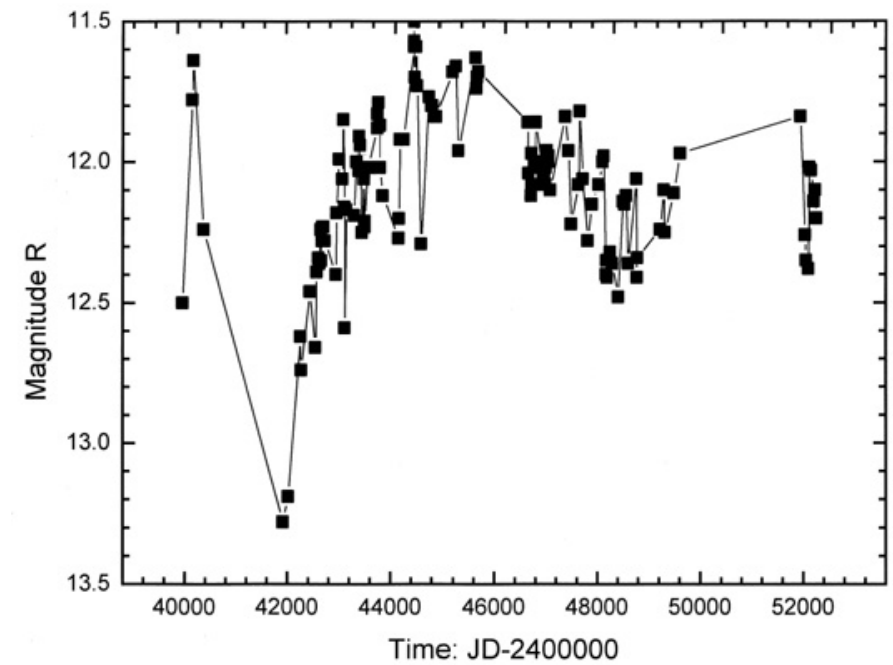

Fig. 8. Observed light curve of V2074 Cyg.

To this list of LPVs we can add the two stars V2074 Cyg and V1983 Cyg from the group of non-periodic stars by Alksnis \& Alksne (1988), which also show long-term changes. The observed light curve of V2074 Cyg is similar to that of a star with $P_{1}<400 \mathrm{~d}$, and there is a partly observed light drop (Figure 8). On the other hand, the star V1983 Cyg additionally to the slow changes underwent several light drops (Figure 9), more similar to those observed for the unusual carbon variable DY Per (Alksnis et al. 1992). 


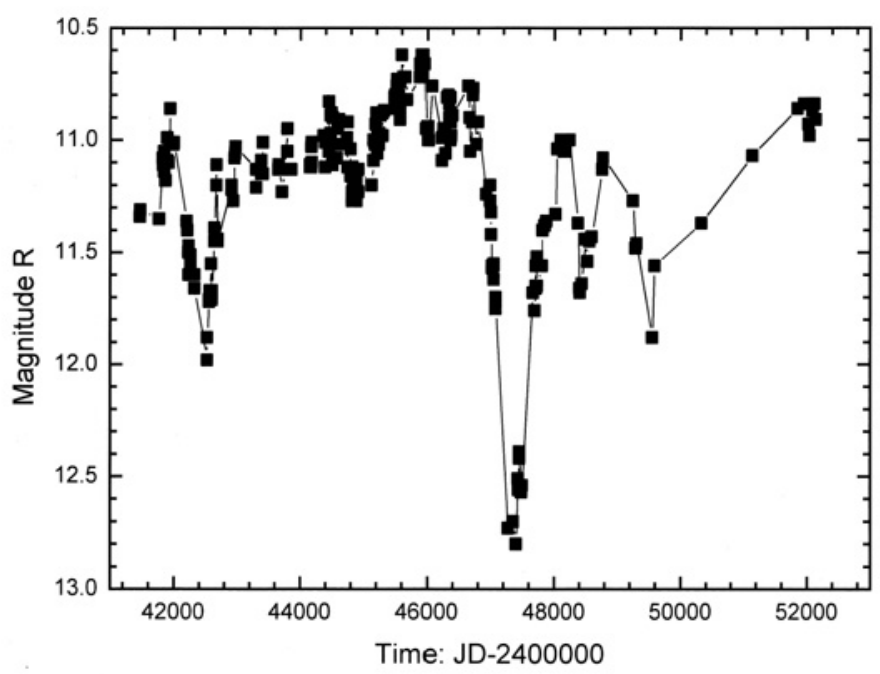

Fig. 9. Observed light curve of V1983 Cyg.

\section{CONCLUSIONS}

Although the number of the stars considered is too small for statistical analysis, we have noticed that there is some correlation between the value of $P_{1}$ and the character of the long-term variation. For the six stars with $P_{1}<400 \mathrm{~d}$ the long-term variations are slow and small, but occasional light drops have been observed for half of them. For the stars with $P_{1}>420 \mathrm{~d}$, more rapid smooth variations with larger range have been observed together with occasional sharper brightenings for three stars. Some other stars show continuous oscillation of a zigzag shape. Two stars considered to be non-periodic also show long-term variations.

ACKNOWLEDGMENTS. I am thankful to all my colleagues, former and present, at my home institute, who during the 30 years, earlier or later, longer or shorter time have taken part in the observations with the Baldone Schmidt telescope, and those who did plate measurements.

\section{REFERENCES}

Alksnis A., Alksne Z. 1977, in Photometric Investigations of Carbon Stars, ed. A. Balklavs, Publ. House Zinātne, Rīga, p. 7 (in Russian)

Alksnis A., Alksne Z. 1988, Carbon Stars in a Field in Cygnus, Zinātne 
Publ. House, Rīga (in Russian)

Alksnis A., Balklavs A., Dzervitis U., Eglitis I., Paupers O., Pundure I. 2001, General Catalog of Galactic Carbon Stars, 3rd ed., CDS Catalogue III $/ 227$

Alksnis A., Larionov V. M., Larionova L. V., Shenavrin V. I. 2002, Baltic Astronomy, 11, 487

Kholopov P. N., Samus N. N., Frolov M. S. et al. 1998, General Catalogue of Variable Stars, 4th ed., CDS Catalogue II/214A

Straižys V. 1977, Multicolor Stellar Photometry, Mokslas Publishers, Vilnius

Whitelock P. A., Feast M. W., Marang F., Overbeek M. D. 1997, MNRAS, 288, 512 